\title{
PERFORMANCE ANALYSIS OF TRANSMIT POWER CONTROL SCHEME IN COGNITIVE RADIO NETWORK
}

\author{
Sandeep Kumar Jain ${ }^{1}$, Y.S. Randhawa ${ }^{2}$ and Kanwaljeet Singh ${ }^{3}$ \\ ${ }^{I}$ Department of Electronics and Communication Engineering, Shri Vaishnav Vidyapeeth Vishwavidyalaya India \\ ${ }^{2}$ Department of Electronics and Communication Engineering, Lyllapur Khalsa College of Engineering, India \\ ${ }^{3}$ Department of Electronics and Communication Engineering, Lovely Professional University, India
}

\begin{abstract}
Cognitive radio is a promising technology which provides efficient radio resource utilization. This paper presents a contextual literature review of the different approaches with their simulation results and identifies the one that best suits the cognitive radio environment. In recent years, a variety of transmit power control algorithms have been proposed for organizing cognitive radio networks. Firstly, we review the two transmit-power control schemes, which are specified in existing research papers, namely fixed and adaptive transmit-power control schemes. Secondly, we proposed transmit-power control schemes based on spectrum sensing side information. In order to achieve better sensing performance, we are employing more number of antennas at secondary user. Due to better sensing performance, we can exercise more accurate control on transmit power of secondary user transmitter.
\end{abstract}

Keywords:

Cognitive Radio, Fixed Power Control, Adaptive Power Control, Sensing Information, Probability of Detection

\section{INTRODUCTION}

With increasing number of users and to get enhanced throughput, requirement of new generation radio system is increasing. Due to this demand, usage of limited spectrum resource which is allocated to various number of users based of fixed assignment policy, is increased. However, it has been found from research that frequencies which are assigned to license users are not fully utilized most of the time. To mitigate the issues of spectrum underutilization and spectrum scarcity [1], cognitive radio [3] [4], has been proposed as promising technology with network architecture [5] [6] in which two types of user, first one is primary users (PU) also known as licensed users having full access to use it, depending on their requirement. While another one is secondary users (SU) or cognitive radio (CR) users, which are not purchasing it but can use without creating interference and interruption to primary user. Cognitive radio has been proposed as an emerging technology, where spectrum sensing and dynamic spectrum access (DSA) [7], techniques provide better resource utilization without creating interference and interruption to primary user. In this access mechanism, the unlicensed users sense the presence of license users by various spectrum sensing techniques [8]-[12]. Based on the result collected by sensing techniques, cognitive users start communication whenever they found that there is no communication going on in that unoccupied spectrum. At that time, when primary users want to start communication, then secondary users have to check the availability of primary users based on high probability and leave the channel or to overcome the interference by changing the level of transmit power of secondary users.
This paper is organized as follows: Initially, literature review have been presented in section 2, then system model for proposed cognitive radio system is given in section 3. Comparisons between existing fixed and adaptive power control schemes in section 4. In section 5, proposed methodology to calculate the shortest distance between PU receiver and SU transmitter is described along with derived mathematical expression for maximum allowable transmit power of SU as a function of shortest distance calculated in section 4. Then in section 6, simulation results are presented and discussed. Finally, conclusions are drawn in section 7 .

\section{LITERATURE REVIEW}

In order to avoid the interference at PU, there are many techniques available in the literature to control the transmit power levels of SU. An orthogonal frequency division multiplexing (OFDM) was considered as a candidate for cognitive radio to avoid the interference by leaving a set of sub-channels unused [13]. Thus, it can provide a flexible spectral shape that fills the spectral gaps without interfering with the licensed users. Beamforming based on the instantaneous channel state information (CSI) [14] from the SU transmitter to the PU receivers allows the SU suppresses the interference to PUs. In one of the schemes for transmit power level control in CRS, we use spectrum sensing side information [15]-[17], wherein we first calculate shortest distance between $\mathrm{CR}$ and $\mathrm{PU}$ receiver then based on the minimum distance transmit power can be controlled. In another approach we employ adaptive power control scheme [18]. By allowing transmit power adaptation at the SU transmitter to maintain a constant output SNR to the SU receiver, this scheme maximizes the output SNR and limits the interference to a primary user within an interference constraint. Power control in cognitive radios under cooperative and non-cooperative spectrum sensing [19]. In non-cooperative spectrum sensing, the SU Tx has its spectrum sensing information (SSI) while in cooperative spectrum sensing, the SU Tx has both its SSI and the SU Rx's SSI. Using the SSIs, power control schemes are designed to either maximize the average data rate or minimize the outage probability of the SU.

\section{PROPOSED SYSTEM MODEL}

Proposed system model for cognitive radio system is shown in Fig.1, where the primary and secondary users coexist in the same area and using same frequency band. Here primary transmitter and receiver are represented by PU Tx and PU Rx respectively; whereas secondary transmitter and receiver are represented by SU 
Tx, and SU Rx respectively. In order to get better sensing information we are considering multiple numbers of antennas denoted by $T$ and $R$ at SU Tx and SU Rx respectively. The circles covered with the radius $R_{d}$ and $R_{p}$ represents, respectively, the decodable region within which the SNR of decodability occurs in the absence of interference to the PU Rx and the protection region within which the PU Rx must be guaranteed successful reception in the presence of SU. Here we are considering received SNR as a proxy for estimating the shortest distance between PU Rx and SU Tx. So, $\Delta$ and $\mu$ (both measured in $\mathrm{dB}$ ), respectively, represent path loss in the region corresponding to $\mathrm{Rd}$ and how much interference above the noise floor the primary system can tolerate i.e. protection margin. There are three types of channels shown in the system model. The user channel is the channel between SUs, sensing channel is the channel from PU transmitter to SU transmitter and the interference channel is the channel from SU transmitter to PU receiver. Symbols $d_{1}$ and $d_{2}$ (both measured in meters) represent the distances between PU Tx - SU Tx and between PU Rx - SU Tx respectively.

In our proposed scheme to control the transmitted power level of SU transmitter for a known transmit SNR of PU, distance $d_{2}$ is required. Due to lack of awareness about the right location of PU and exact channel information, the calculation of distance $d_{2}$ is very difficult. Therefore, as shown in the system model, the path loss between PU Tx and SU Tx is represented by $(\Delta+\Psi) \mathrm{dB}$. Now, if transmit power of the SU depends on the path loss $(\mu+\Psi)$ $\mathrm{dB}$ between PU Rx and SU Tx. Then, the transmit power control problem is essentially converted to the problem of evaluating the path loss due to $d_{1}$ for a given $\mu$ and $\Delta$. We take the path loss exponent between PU Tx - PU Rx as $\alpha_{1}$ and between SU Tx - PU $\mathrm{Rx}$ as $\alpha_{2}$, which are usually constant in the range $2 \sim 5$.

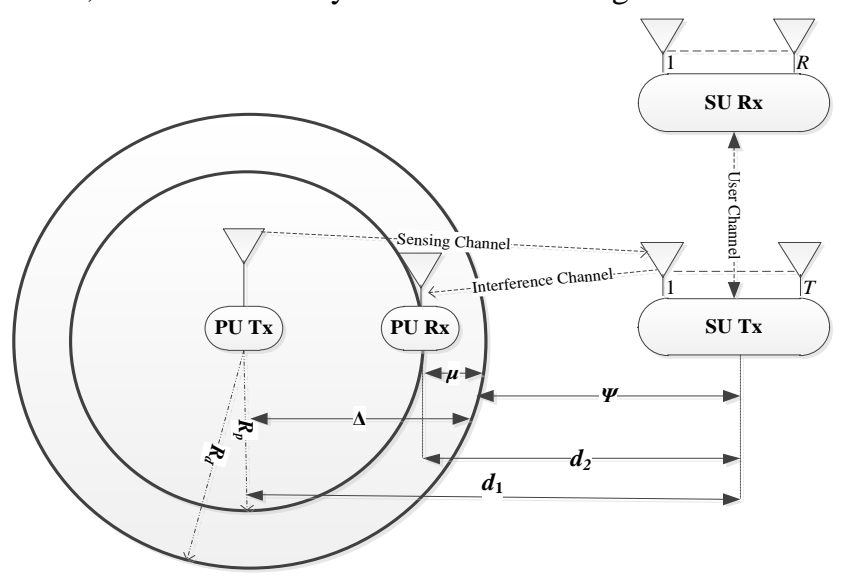

Fig.1. Proposed System Model

\section{TRANSMIT POWER CONTROL SCHEMES}

In order to avoid interference at primary user by cognitive user, number of power control techniques has been given in various research paper. Here we are comparing fixed and adaptive power control scheme and the proposed a scheme with better control on transmitting power based on distance calculation between PU Rx and SU Tx.

\subsection{FIXED POWER CONTROL SCHEME}

Fixed-power control scheme [18] provide a straightforward way to control the transmit power of secondary user based on given interference constraints of PUs specified by two parameters $\{\eta, \xi\}$. As shown in Fig.2, the SU transmitter first perform initialization such as loading software for DSA. Then, to identify the control channel spectrum sensing performs and SU transmitter receives the interference constraint of PUs. In step 4, while satisfying these interference constraints, SU transmitter calculates the maximum fixed transmit power. Once this fixed transmit power is determined, SU transmitter performs spectrum sensing by using the energy detection (ED) with selective combining (SC) in step 5. In step 6, the SU starts transmission, if spectrum opportunity is found.

In order to calculate the maximum fixed transmit power at secondary user the expression for the distribution of the instantaneous INR in the interference channel as a function of the constant transmit power is required. We use this expression with the detection probability and obtain the maximum fixed transmit power, which allows us to further calculate the BER for the SU transmission.

According to spectrum sensing information, SUs start transmission once the unoccupied spectrum is found. This opportunistic transmission by SUs will interfere with PUs if the former fails to detect the presence of the latter's signals, i.e. misdetection (MD), and starts the transmission in the same frequency band with a large transmit power such that the INR exceeds the threshold $\eta$ given by the interference constraint. We can express the probability of having the instantaneous INR larger than $\eta$ in the case of MD as [18]

$$
\operatorname{Pr}\left\{I_{n}>\eta \mid M D\right\}=\left\{1-P_{d . s c}\left(T, N_{0}\right)\right\}\left\{1-F_{I_{n}}(\eta)\right\}
$$

where, $P_{d . s c}\left(T, N_{0}\right)$ is the detection probability by the ED with SC in a Rayleigh fading channel and $F_{I_{n}}(\eta)$ is the cumulative distribution function (CDF) of instantaneous INR can be given as

$$
\begin{aligned}
& P_{d, s c}\left(T, N_{0}\right)=2 T \sum_{n=0}^{T-1}(-1)^{n}\left(\begin{array}{c}
T-1 \\
n
\end{array}\right) \frac{1}{p^{2}} \exp \left(\frac{-b^{2}}{2}\right) \times \\
& \left\{\frac{p^{2}+a^{2}}{a^{2}}\right)^{N_{0}-1}\left[\begin{array}{l}
\exp \left(\frac{1}{2} b^{2} \frac{a^{2}}{p^{2}+a^{2}}\right)- \\
\left.\left.\sum_{m=0}^{N_{0}-2} \frac{1}{m !}\left(\frac{1}{2} b^{2} \frac{a^{2}}{p^{2}+a^{2}}\right)^{m}\right]+\sum_{m=0}^{N_{0}-2} \frac{1}{m !}\left(\frac{b^{2}}{2}\right)^{m}\right\}
\end{array}\right.
\end{aligned}
$$

where, symbols $p, a$ and $b$ are defined in terms of number of samples $\left(N_{0}\right)$ detection threshold of ED $\left(E_{t h}\right)$, average sensing SNR $\left(\Gamma_{s}\right)$ and standard deviation of the receiver noise $\left(\sigma_{s}\right)$ at SU Tx.

$$
\begin{gathered}
p=\sqrt{2(n+1)} \\
a=\sqrt{2 \Gamma_{S} N_{0}} \\
b=\frac{\sqrt{2 E_{t h}}}{\sigma_{z}}
\end{gathered}
$$

In order to calculate the detection probability by using Eq.(2), we require the value of decision threshold $E_{t h}$ for a given false 
alarm rate, $P_{f .} E_{t h}$ can be determined using Eq.(3) given below in which $\Gamma(.,$.$) and \Gamma($.$) denotes incomplete gamma function and$ gamma function respectively.

$$
\begin{gathered}
P_{f}\left(E_{t h}, N_{0}, \sigma_{z}\right)=\frac{\Gamma\left(N_{0}, \frac{E_{t h}}{2 \sigma_{z}^{2}}\right)}{\Gamma\left(N_{0}\right)} \\
F_{I_{n}}(\eta)=\frac{\Gamma\left(\left(\frac{\eta T}{p_{t} \Gamma_{S}}\right), T\right)}{\Gamma(T)}
\end{gathered}
$$

where, $I_{n}$ is instantaneous INR.

It is clear from the above Eq.(1) that $\operatorname{Pr}\left\{I_{n}>\eta \mid M D\right\}$ is a function of $P_{t}$, SU transmitter can calculate the maximum $P_{t}$ such that $\operatorname{Pr}\left\{I_{n}>\eta \mid M D\right\}<\xi$ according to the given interference constraint in step 4.

The BER of the SU transmission for the instantaneous output SNR is given by Eq.(5), with argument fixed transmit power $P_{t}$ is applied at SU transmitter as [18]

$$
P_{e}=\left(\frac{(1-\mu)}{2}\right)^{T R} \sum_{m=0}^{T R-1}\left(\begin{array}{c}
T R-1+m \\
m
\end{array}\right)\left(\frac{1+\mu}{2}\right)^{m}
$$

where,

$$
\mu=\sqrt{\frac{P_{t} \Gamma_{u}}{T+P_{t} \Gamma_{u}}}
$$

\subsection{ADAPTIVE POWER CONTROL SCHEME}

The adaptive power control scheme [18] adjusts the transmit power based on the varying channel status of the user channel while satisfying the interference constraint. Adaptive power control scheme allows transmit power adaptation at the secondary user (SU) transmitter to maintain a constant output SNR to the SU receiver. This scheme maximizes the output SNR and limits the interference to a primary user (PU) within an interference constraint of PUs specified by two parameters $\{\eta, \xi\}$.

As shown in Fig.2, the SUs perform initialization in the first step. Then, performs spectrum sensing to identify the control channel and SU transmitter receives the interference constraint of PUs. In step 4, while satisfying these interference constraints, SU transmitter calculates the maximum constant output SNR at SU receiver. The goal of the adaptive-power control scheme is to maximize the constant output SNR at SU receiver while limiting the interference to the PU receiver within the interference constraint. Spectrum opportunity is found by spectrum sensing performs by SU transmitter in step 5. Once spectrum opportunity is found, SU transmitter sends the calculated maximum output SNR as a target level to SU receiver in step 6.

Then, based on channel condition and quality of services, SU receiver sends command to SU transmitter to vary the transmit power to maintain the output SNR at that target level. Subsequently, the SU starts data transmission in next step.

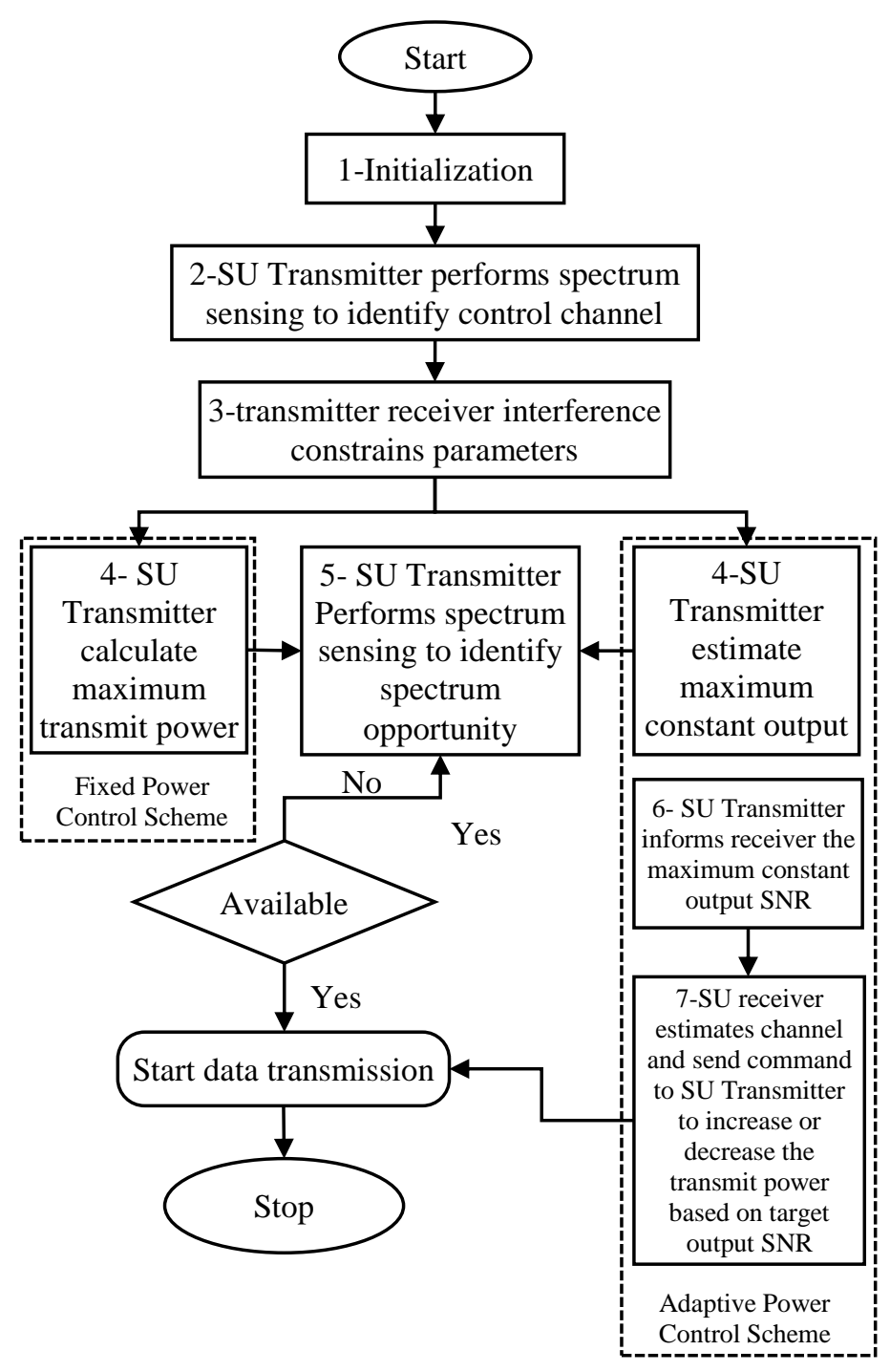

Fig.2. Flow chart diagram for fixed and adaptive power control

In order to calculate the maximum output SNR as a target level to SU receiver, at SU transmitter the expression for the distribution of the instantaneous INR in the interference channel as a function of the constant output SNR at SU receiver is required. We use this expression with the detection probability and obtain the maximum output SNR at SU receiver.

Similarly as in fixed power control scheme, we can express the probability of having the instantaneous INR larger than $\eta$ in the case of MD as [18],

$$
\operatorname{Pr}\left\{I_{n}>\eta \mid M D\right\}=\left\{1-P_{d . s c}\left(T, N_{0}\right)\right\}\left\{1-F_{I_{n}}^{\prime}(\eta)\right\}
$$

where, $F_{I_{n}}^{\prime}(\eta)$ is the cumulative distribution function (CDF) of instantaneous INR in adaptive power control scheme can be given as:

$$
\begin{aligned}
& F_{I_{n}}^{\prime}(\eta)=\int_{0}^{\eta} F_{I_{n}}(x) d x \\
& =2 F_{1}\left(T, T+T R ; T+1 ;-\frac{\eta}{\alpha \Gamma_{S}}\right)\left(\frac{\eta}{\alpha \Gamma_{S}}\right)^{T} \times \frac{\Gamma(T R+T)}{T \Gamma(T+1) \Gamma(T R)}
\end{aligned}
$$

where, $2 F_{1}(., . ; . ;$.$) is the hyper-geometric function [21].$ 
As shown above in Eq.(6), $\operatorname{Pr}\left\{I_{n}>\eta \mid M D\right\}$ is a function of $\alpha$, SU transmitter can calculate the maximum constant output SNR such that $\operatorname{Pr}\left\{I_{n}>\eta \mid M D\right\}<\xi$ satisfy interference constraint. We can calculate the BER as [18]:

$$
P_{e}=Q\left(\sqrt{2 \alpha \Gamma_{u}}\right)
$$

\subsection{POWER CONTROL BASED ON SPECTRUM SENSING SIDE INFORMATION}

Power control approach in cognitive radio systems based on spectrum sensing side information [16] [17] consists of two steps. Firstly, the shortest distance between a licensed receiver and a cognitive radio is derived from the spectrum sensing side information. Then, the transmit power of the cognitive radio is determined based on this shortest distance to guarantee a quality of service for the licensed user. Because the worst case is considered in this approach where the cognitive radio is the closest to the licensed user, the proposed power control approach can be applied to the licensed user in any location.

In this chapter, we proposed distance based an efficient transmit power control scheme in cognitive radio with multiple antennas at SUs, which allows SU transmitters to aggressively increase the level of their transmit powers while still guaranteeing an acceptable level of aggregate interference at the PU receivers. This proposed scheme is based on the modification of existing power control scheme based on spectrum sensing side information. This modification gives more accuracy to control transmit power in comparison of existing one. In order to estimate allowable transmit power for SU we considered received SNR as a proxy to determine the shortest distance between SU transmitter and PU receiver.

We have also derived a mathematical model for calculating shortest distance in terms of probability of detection. We consider the coexistence between an unlicensed user i.e. cognitive user and a licensed user in order to enhance the spectrum efficiency.

\section{PROPOSED METHODOLOGY TO CALCULATE DISTANCE BETWEEN PU TRANSMITTER AND SU TRANMITTER}

In this section we discuss our proposed methodology to calculate the distance $d_{l}$ between PU Tx and SU Tx which will be used to control and determine maximum allowable transmit power at SU Tx while still guaranteeing decodability of PU signal at PU Rx in the protected region.

\subsection{SPECTRUM SENSING}

Spectrum sensing is one of the most vital issues of cognitive radio technology as it should be firstly performed before allowing unlicensed users to access an unoccupied licensed band, to ensure the efficient utilization of the spectrum without disturbing the quality of service of the primary user. There are many techniques for spectrum sensing available in literature [10] - [12].

In order to avoid the interference at the PU Rx, the SU should sense the spectrum opportunity. Due to simplicity and extremely low processing load, we use energy detection with selective combining for sensing the available unoccupied frequency band.
If $y(t)$ is the received signal at the SU Tx, $x(t)$ is the transmitted signal from PU Tx, $n(t)$ is the zero-mean additive white Gaussian noise (AWGN) with the variance $\sigma^{2}$ and $h$ denotes the Rayleigh fading channel coefficient. The objective of spectrum sensing is to decide between the following two hypotheses:

When the PU Tx is not active, which is referred to as hypothesis $H_{0}$, the received signal at the $i^{\text {th }}$ antenna of SU Tx is given by:

$$
y(t)=n(t) \text {. }
$$

When the PU Tx is active, which is referred to as hypothesis $H_{1}$, the signal received from the PU Tx at the $i^{\text {th }}$ antenna of SU Tx can be written as:

$$
y(t)=\sqrt{G_{S}} h x(t)+n(t)
$$

where, $G_{s}$ represent path loss of sensing channel.

Based on these two hypotheses $H_{0}$ and $H_{1}$ average probability of false alarm, probability of detection and probability of missing over Rayleigh fading channel can be determined respectively as given,

$$
\begin{gathered}
P_{f}=E\left[\operatorname{prob}\left\{H_{1} / H_{0}\right\}\right] \\
P_{d}=E\left[\operatorname{prob}\left\{H_{1} / H_{1}\right\}\right] \\
P_{m}=E\left[\operatorname{prob}\left\{H_{0} / H_{1}\right\}\right]=1-P_{d}
\end{gathered}
$$

where, $E$ represent expectation operator.

\subsection{RELATION ESTABLISHMENT BETWEEN SNR AND DISTANCE}

To calculate distance $d_{2}$ for controlling the transmit power of SU is very difficult because we do not know the exact location of PU as well as exact channel state information. As mentioned in our system model, discussed in section 2 , the problem of finding $d_{2}$, is essentially the problem of finding $d_{1}$. The distance, $d_{1}$, can be estimated by measuring the local SNR of the PU Tx at SU Tx with the help of pilot signal as well as sensing SNR. In this subsection, we have derived the relationship between sensing SNR and distance $\left(d_{1}\right)$. The path loss due to distance $d_{1}$ between PU Tx and SU Tx is given by [15],

$$
P L=-10 \log \left(d_{1}^{\left(-\alpha_{1}\right)}\right) d B
$$

In term of average sensing SNR $\left(\Gamma_{S}\right)$ and transmit SNR of PU $\operatorname{Tx}\left(\Gamma_{t}\right)$ above Eq.(11) can be written as,

$$
P L=10 \log \left(\Gamma_{t}\right)-10 \log \left(\Gamma_{S}\right)
$$

From Eq.(11) and (12) average SNR of sensing channel can be represented in term of transmit SNR of PU Tx and distance $d_{1}$ as

$$
\Gamma_{S}=\Gamma_{t} d_{1}^{\left(-\alpha_{1}\right)}
$$

\subsection{DISTANCE BETWEEN PU TX AND SU TX}

Now substitute Eq.(13) in Eq.(2), we get detection probability as a function of distance $d_{1}$. 


$$
\begin{aligned}
P_{d, s c}^{\prime}\left(T, N_{0}\right) & =2 T \sum_{n=0}^{T-1}(-1)^{n}\left(\begin{array}{c}
T-1 \\
n
\end{array}\right) \frac{1}{p^{2}} \exp \left(\frac{-b^{2}}{2}\right) \times \\
& \left\{\begin{array}{l}
\left(\frac{p^{2}+2\left(\Gamma_{t} d_{1}^{\left(-\alpha_{1}\right)}\right) N_{0}}{2\left(\Gamma_{t} d_{1}^{\left(-\alpha_{1}\right)}\right) N_{0}}\right)^{N_{0}-1} \\
{\left[\begin{array}{l}
\exp \left(\frac{1}{2} b^{2} \frac{2\left(\Gamma_{t} d_{1}^{\left(-\alpha_{1}\right)}\right) N_{0}}{p^{2}+2\left(\Gamma_{t} d_{1}^{\left(-\alpha_{1}\right)}\right) N_{0}}\right)- \\
\sum_{m=0}^{N_{0}-2} \frac{1}{m !}\left(\frac{1}{2} b^{2} \frac{2\left(\Gamma_{t} d_{1}^{\left(-\alpha_{1}\right)}\right) N_{0}}{p^{2}+2\left(\Gamma_{t} d_{1}^{\left(-\alpha_{1}\right)}\right) N_{0}}\right)^{m}
\end{array}\right]} \\
+\sum_{m=0}^{N_{0}-2} \frac{1}{m !}\left(\frac{b^{2}}{2}\right)^{m} \\
P_{m}=1-P_{d, s c}^{\prime}\left(T, N_{0}\right)=1-f\left(d_{1}\right)
\end{array}\right.
\end{aligned}
$$

By using the above equation, we can calculate distance $d_{1}$ between PU Tx and SU Tx for any particular value of detection probability, $P_{d, S C}$.

\section{PROPOSED TRANSMIT POWER CONTROL SCHEME AS A FUNCTION OF DISTANCE D1}

In this section, we derived mathematical expression to determine the maximum allowable transmit power at SU Tx while still guaranteeing decodability of PU signal at PU Rx in protected region.

Let $P_{p}$ and $P_{s}$ are the transmit powers of primary and secondary transmitters, respectively, with their corresponding received powers at PU Rx denoted by $P_{P}^{\prime}$ and $P_{S}^{\prime}$, on the edge of the protected region. If noise power at PU Rx denoted by $\sigma_{w}^{2}$, then guaranteed decodability of PU Tx signal at PU Rx having decodable SNR $\left(\Gamma_{d e c}\right)$ can be expressed as:

$$
\begin{gathered}
10 \log \left(\frac{P_{P}^{\prime}}{P_{S}^{\prime}+\sigma_{w}^{2}}\right) \geq \Gamma_{d e c} \\
\frac{P_{P}^{\prime}}{P_{S}^{\prime}+\sigma_{w}^{2}} \geq 10^{\Gamma_{\text {dec }} / 10}
\end{gathered}
$$

We can express $P_{P}^{\prime}$ within protected region in terms of SNR as,

$$
10 \log \left(\frac{P_{P}^{\prime}}{\sigma_{z}^{2}}\right)=\Gamma_{d e c}+\mu
$$

Now from Eq.(20) we see that the secondary user must guarantee:

$$
P_{S}^{\prime} \leq \sigma_{w}^{2}\left(10^{\frac{\mu}{10}}-1\right)
$$

In order to successful reception of PU Tx signals at PU Rx, a fundamental constraint for any cognitive system is given by above
Eq.(17). The allowable maximum transmits power for secondary user in terms of distance $d_{2}$ between PU Rx and SU Tx, thus, can be expressed as:

$$
P_{S} \leq d_{2}^{\alpha_{2}} \sigma_{w}^{2}\left(10^{\frac{\mu}{10}}-1\right)
$$

Now, by using received SNR as a proxy for calculating the value of distance $d_{2}$, transmit power of secondary transmitter can be controlled by using Eq.(22) given below [13]:

$$
\begin{aligned}
10 \log \left(\frac{P_{S}}{\sigma_{w}^{2}}\right) \leq & \frac{\alpha_{2}}{\alpha_{1}} \Delta+10 \log \left(10^{\mu / 10}-1\right)+ \\
& 10 \alpha_{2} \log \left\{\left(10^{\mu / 10}\right)^{1 / \alpha_{1}}-\left(10^{-\mu / 10}\right)^{1 / \alpha_{1}}\right\}
\end{aligned}
$$

The first term describes, that how far a PU Rx can travel from the PU Tx and still decode the signal. The second term represents how tolerant the protected primary receivers are to interference. The final term represents how far the secondary transmitter is from the protected receivers.

$$
\begin{gathered}
10 \log \left(\frac{P_{S}}{\sigma_{w}^{2}}\right) \leq \frac{\alpha_{2}}{\alpha_{1}} \Delta+10 \alpha_{2} \log \left(10^{\mu / 10}\right)^{1 / \alpha_{1}}-\left(10^{-\mu / 10}\right)^{1 / \alpha_{1}}+ \\
10 \log \left(10^{\mu / 10}-1\right)+10 \log \left(\sigma_{w}^{2}\right) \\
P_{S}^{\max } \mid d B=g(\psi) \\
P_{S}^{\max } \mid d B=g(P L-\Delta) \\
P_{S}^{\max } \mid d B=g\left(-10 \log \left(d_{1}^{\left(-\alpha_{1}\right)}\right)-\Delta\right)
\end{gathered}
$$

where, $P_{S}^{\max }$ denotes the maximum value of transmit power used at SU transmitter and, as shown above in Eq.(26), this maximum allowable transmit power at SU Tx is controlled on the basis of calculated distance $d_{1}$ between PU Tx and SU Tx.

\section{SIMULATION RESULTS AND DISCUSSIONS}

In this section, we present and discuss the simulation results of the fixed, adaptive transmit power and proposed transmit power control scheme. The simulations have been carried out in MATLAB. We consider the situation where there are $T$ antennas at SU Tx observing independent Rayleigh fading with equal average SNRs in the sensing channel. Furthermore, the ED is performed using the signals from the selected antenna to determine the presence or absence of the PU signals with the parameters as shown in Table.1 have been considered.

Table.1. Simulation parameters

\begin{tabular}{|c|c|}
\hline Name of the parameter & Value \\
\hline $\begin{array}{c}\text { Path loss in the region } \\
\text { corresponding to } R_{d}\end{array}$ & $\Delta=100 \mathrm{~dB}$ \\
\hline Protection margin. & $\mu=1 \mathrm{~dB}$ \\
\hline Transmit SNR of PU & $\Gamma_{t}=100 \mathrm{~dB}$ \\
\hline False alarm rate & $P_{f}=0.01$ \\
\hline Number of Samples & $N_{0}=5$ \\
\hline
\end{tabular}




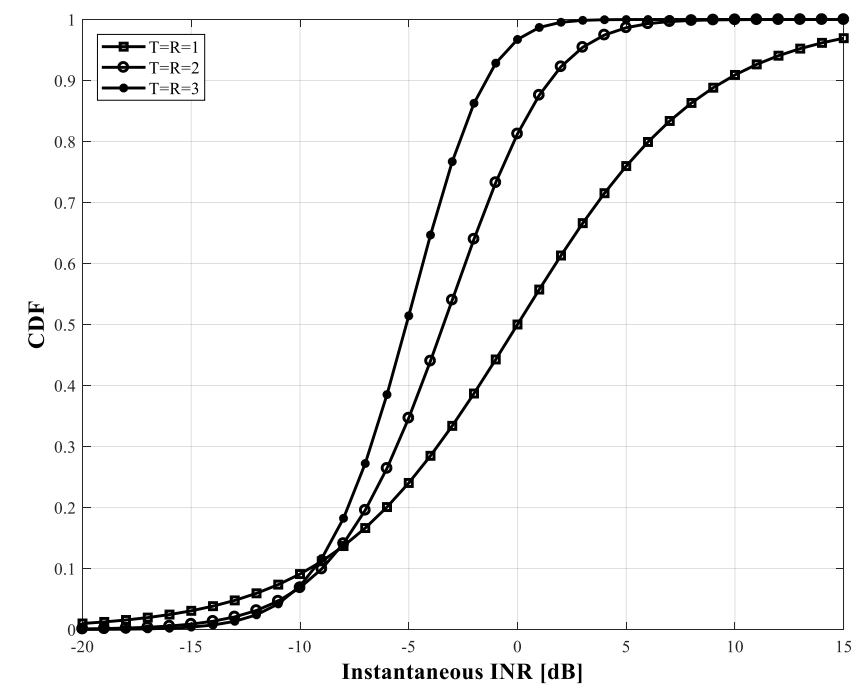

Fig.3. CDF of the instantaneous INR at the PU when adaptive power control is employed at the SU transmitter for different numbers of antennas $\left(\alpha=1\right.$ and $\left.\Gamma_{S}=0 \mathrm{~dB}\right)$

In Fig.3, we examine the distribution of the instantaneous INR at the PU for the adaptive power control scheme for $\alpha=1$ and $\Gamma_{u}$ $=0 \mathrm{~dB}$ when the number of antennas at SUs changes. It is seen that as the number of antennas at SU transmitter increases, the CDF curves move to the left. This indicates a potential reduction in interference to the PU because of better sensing performance with multiple antennas at SU transmitter. For example, the probability of having the instantaneous INR smaller than $0 \mathrm{~dB}$ increases from 0.5 to 0.97 .

The Fig.4 shows comparison between fixed and adaptive power control scheme based on BER performance for different average SNRs of user channel. We assume that the average SNR of the sensing channel and the average INR of the interference channel are equal to $0 \mathrm{~dB}$, i.e., $\Gamma_{s}=0 \mathrm{~dB}$, and $\Gamma_{1}=0 \mathrm{~dB}$.

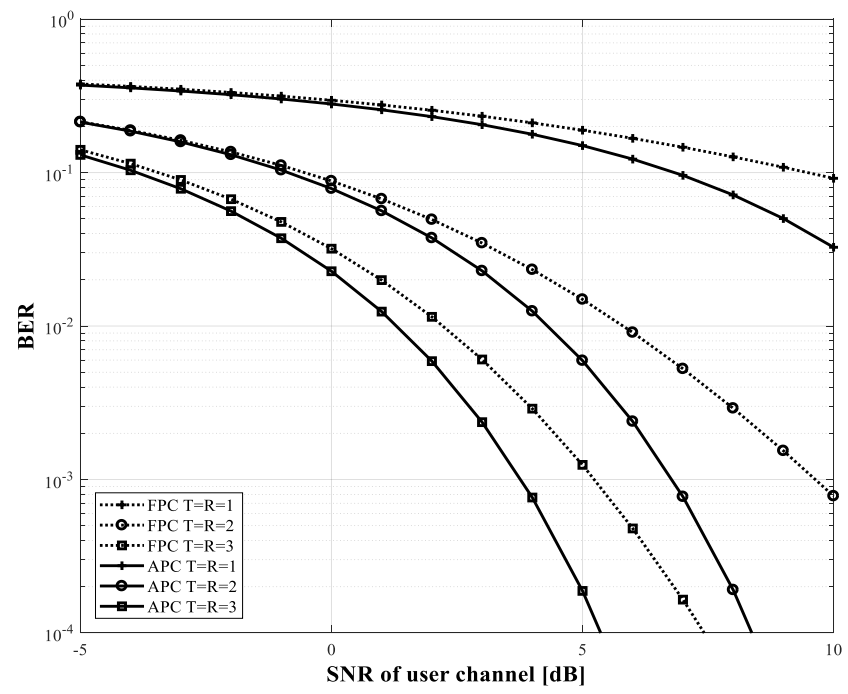

Fig.4. Analytical BERs for the SU transmissions with BPSK signaling, when the fixed and adaptive power control schemes are employed $\left(\Gamma_{S}=0 \mathrm{~dB}\right.$ and $\left.\Gamma_{i}=0 \mathrm{~dB}\right)$

It is seen that the adaptive power control scheme outperforms the fixed power control scheme for different numbers of antennas.
For example, the adaptive power control scheme achieves $3 \mathrm{~dB}$ gain in average SNR of user channel while keeping simulation parameters as $\mathrm{T}=\mathrm{R}=2$ at the $\mathrm{BER}=10^{-3}$.

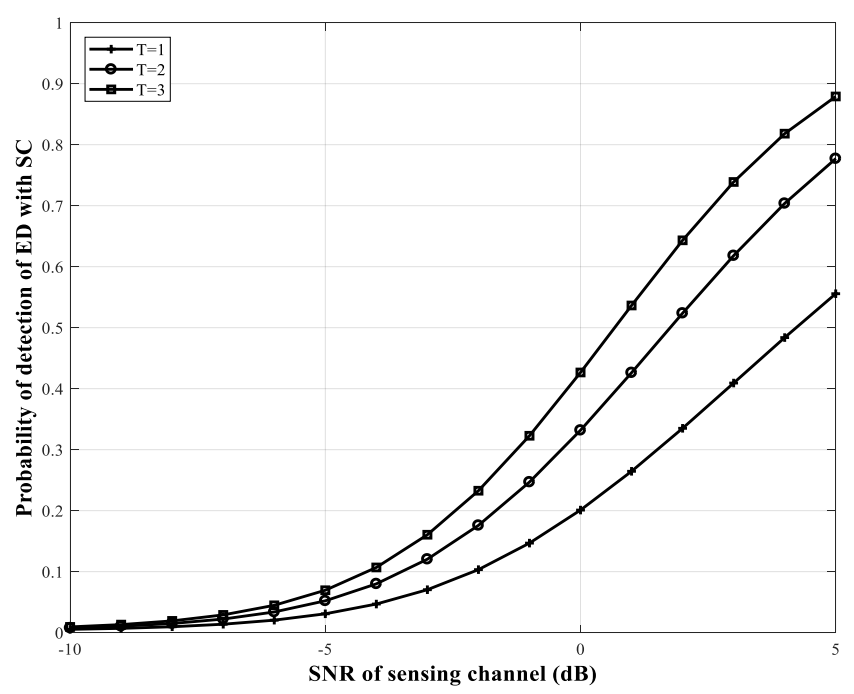

Fig.5. Detection probability using ED with SC in a Rayleigh fading channel. $\left(P_{f}=0.01\right.$ and $\left.N_{0}=5\right)$

The Fig.6 presents the probability of missing as a function of path loss due to distance for different values of path loss exponent, for $\alpha_{1}$ the case of a single antenna at SU Tx. It is seen that the probability of missing increases with increased path loss due to distance between PU Tx and SU Tx. Also, for a particular path loss, a larger path loss exponent will result into increased probability of missing. This is because of the fact that larger the path loss exponent; worse is the wireless communication environment.

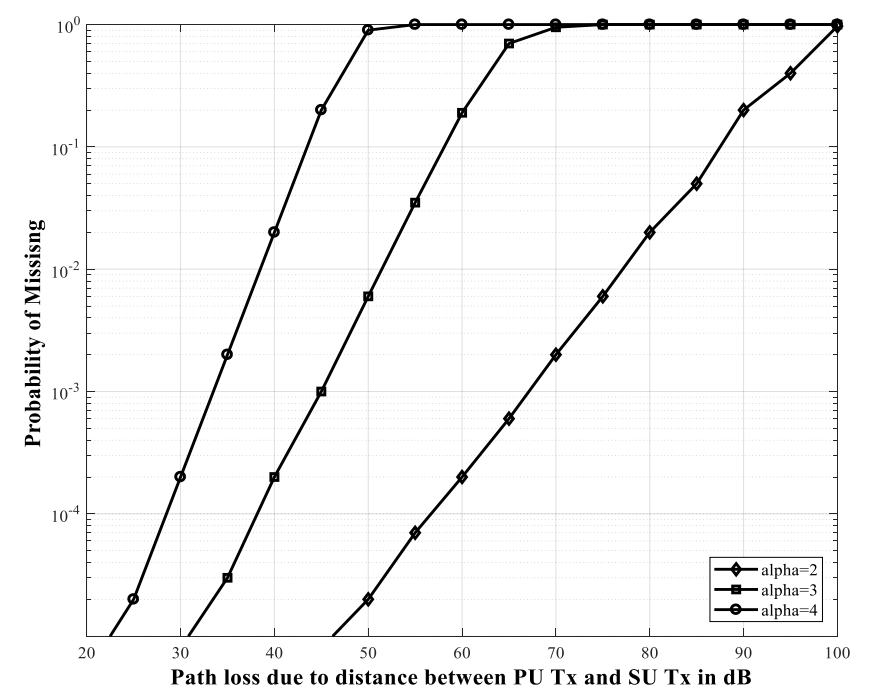

Fig.6. Probability of missing $\left(P_{m}\right)$ versus path loss due to distance $\left(d_{1}\right)$ for different value of path loss exponent between PU Tx and SU Tx 


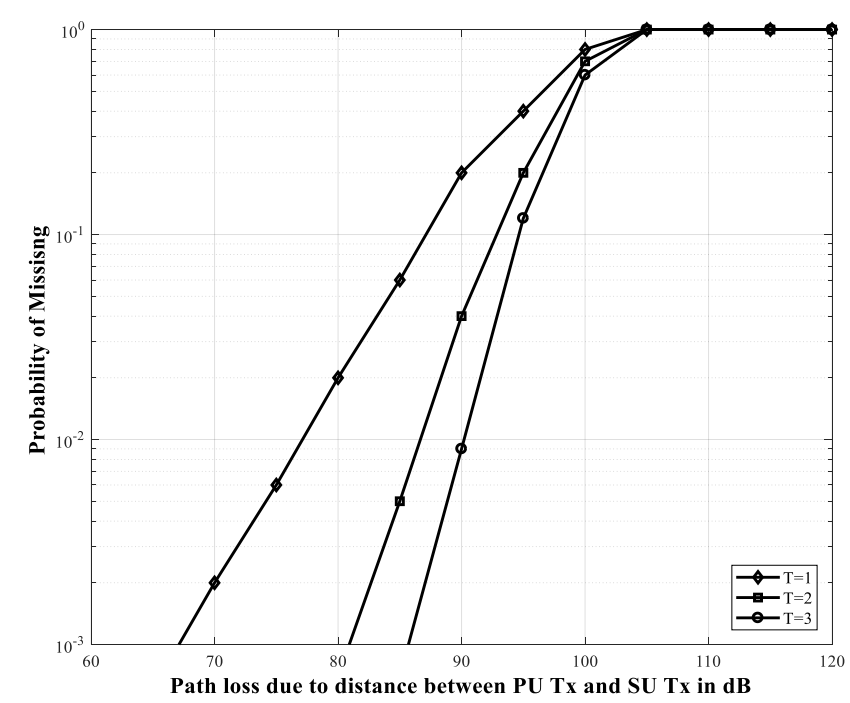

Fig.7. Probability of missing $\left(P_{m}\right)$ versus path loss due to distance $\left(d_{1}\right)$

In Fig.7, we have plotted the probability of missing as a function of path loss due to distance, for different number of antennas at SU Tx, while keeping path loss exponent $\alpha_{1}=2$. Result shows that when cognitive radio is experiencing heavy path loss from the PU Tx, the probability of missing is increased. Also, as is evident, for a particular path loss, the probability of missing can be reduced by employing more number of antennas at SU Tx.

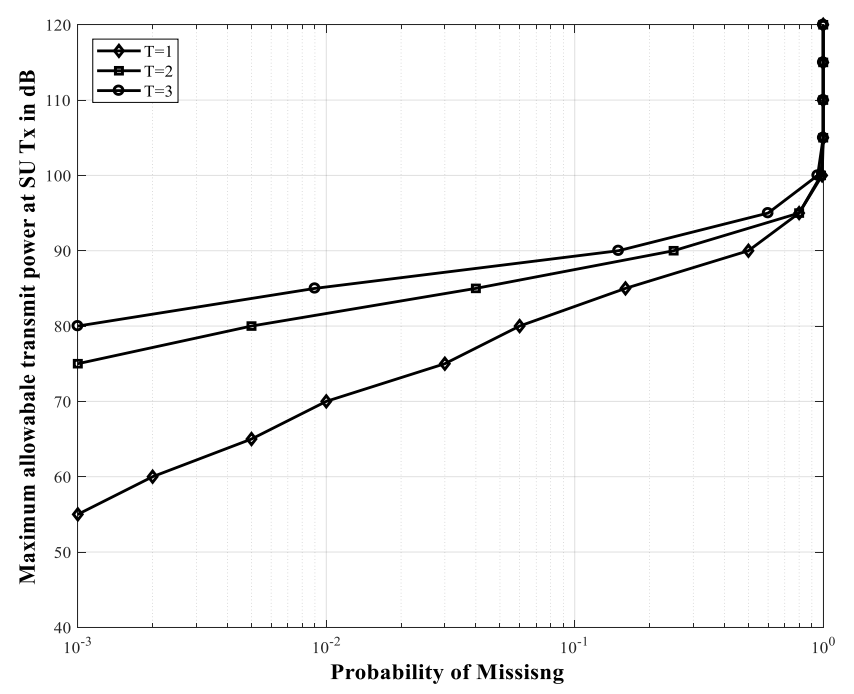

Fig.8. Maximum secondary transmitter power $\left(P_{S}^{\max }\right)$ versus Probability of missing (Pm)

Finally, Fig.8 demonstrates the relationship between maximum allowable transmit power $P_{S}^{\max }$ and probability of missing $P_{m}$ for different number of antenna at SU Tx. The Fig.8 also emphasizes the fact that higher probability of detection can be achieved by employing more number of antennas at SU Tx. It is also obvious that better the probability of detection, more accurate will be our power control scheme.

\section{CONCLUSION}

In this paper, we considered the situation where primary user (PU) shares spectrum with secondary user (SU) i.e. cognitive radio. We have proposed a distance based efficient transmit power control scheme which calculate maximum allowable transmit power at SU transmitter while guarantying decodable signal of PU transmitter at PU receiver in presence of cognitive radio. In order to calculate desired distance we have also derived a mathematical model for probability of detection in terms of distance between PU transmitter and SU transmitter which includes the location information of primary user indirectly.

This scheme has shown that we can achieve better sensing performance by employing more number of antennas at SU Tx. Because of better sensing performance, we can exercise more accurate control on transmit power of SU Tx. In this work, though, we considered worst case scenario where PU Rx is at shortest distance to SU Tx; however, the proposed scheme is equally applicable to maintain a Quality-Of-Service for the PU by limiting the interference generated by SU Tx, at any location.

\section{REFERENCES}

[1] W. Ren, Q. Zhao and A. Swami, "Power Control in Cognitive Radio Networks: How to Cross a Multi-Lane Highway", IEEE Journal on Selected Areas in Communications, Vol. 27, No. 7, pp. 1283-1296, 2009.

[2] Daniel Zwillinger, "Table of Integrals, Series and Products", $8^{\text {th }}$ Edition, Academic Press, 2014.

[3] J. Mitola and G. Q. Maguire, "Cognitive Radio: Making Software Radio more Personal", IEEE Personal Communications, Vol. 6, No. 4, pp. 13-18, 1999.

[4] S. Haykin, "Cognitive Radio: Brain-Empowered Wireless Communications", IEEE Journal on Selected Areas in Communications, Vol. 23, No. 2, pp. 201-220, 2005.

[5] J. Mitola, "Cognitive Radio: An Integrated Agent Architecture for Software Defined Radio", PhD Dissertation, Doctor of Technology, Royal Institute of Technology, 2000.

[6] Sandeep Kumar Jain, Pritesh Kumar Jain and Kanwaljeet Singh, "Cognitive Radio as Encouraging Technology for Efficient Spectrum Resource Utilization", International Journal of Computer Technology and Applications, Vol. 9, No. 41, pp. 5-11, 2016.

[7] Q. Zhao and B.M. Sadler, "A Survey of Dynamic Spectrum Access: Signal Processing, Networking, Regulatory Policy", IEEE Signal Processing Magazine, Vol. 24, No. 3, pp. 7989, 2007.

[8] R. Tandra, M. Mishra and A. Sahai, "What is a Spectrum Hole and What Does it Take to Recognize One?", Proceedings of the IEEE, Vol. 97, No. 5, pp. 824-848, 2009.

[9] R.V. Prasad et al., "Cognitive Functionality in Next Generation Wireless Networks: Standardization Efforts", IEEE Communications Magazine, Vol. 46, No. 4, pp. 72-78, 2008.

[10] T. Yucek and H. Arslan, "A Survey of Spectrum Sensing Algorithms for Cognitive Radio Applications", IEEE Communications Surveys and Tutorials, Vol. 11, No. 1, pp. 116-130, 2009. 
[11] S. Haykin, D.J. Thomson and J.H. Reed, "Spectrum Sensing for Cognitive Radio", Proceedings of the IEEE, Vol. 97, No. 5, pp. 849-877, 2010.

[12] Y. Zeng, Y.C. Liang, A.T. Hoang and R. Zhang, "A Review on Spectrum Sensing Techniques for Cognitive Radio: Challenges and Solutions", EURASIP Journal on Advances in Signal Processing, Vol. 2010, No. 1, pp. 1-15, 2010.

[13] T. Weiss, J. Hillenbrand, A. Krohn and F. K. Jondral, "Mutual Interference in OFDM-based Spectrum Pooling Systems", Proceedings of IEEE 59 ${ }^{\text {th }}$ Vehicular Technology Conference, pp. 1873-1877, 2004.

[14] R. Zhang and Y.C. Liang, "Exploiting Multi-Antennas for Opportunistic Spectrum sharing in Cognitive Radio Networks", IEEE Journal of Selected Topics in Signal Processing, Vol. 2, No. 1, pp. 88-102, 2008.

[15] N. Hoven and A. Sahai, "Power Scaling for Cognitive Radio", Proceedings of International Conference on Wireless Networks, Communications and Mobile Computing, pp. 250-255, 2005.
[16] Karama Hamdi, Wei Zhang and Khaled Ben Letaief, "Power Control in Cognitive Radio Systems Based on Spectrum Sensing Side Information", Proceedings of IEEE International Conference on Communications, pp. 15-19, 2007.

[17] Sandeep Kumar Jain, Manoranjan Rai Bharti and Amardip Kumar, "Distance based an Efficient Transmit Power Control Scheme in Cognitive Radio System with Multiple Antennas", International Journal of Computer Applications, Vol. 72, No. 21, pp. 32-37, 2013.

[18] Chen Sun, Yohannes D.Alemseged, Ha Nguyen Tran and Hiroshi Harada, "Transmit Power Control for Cognitive Radio Over a Rayleigh Fading Channel", IEEE Transactions on Vehicular Technology, Vol. 59, No. 4, pp. 1847-1857, 2010.

[19] Edward C.Y. Peh, Ying Chang Liang, Yong Liang Guan and Yonghong Zeng, "Power Control in Cognitive Radios under Cooperative and Non-Cooperative Spectrum Sensing", IEEE Transactions on Wireless Communications, Vol. 10, No. 12, pp. 4238-4248, 2011. 